\title{
Chemical freeze-out parameters via a nonperturbative QCD approach
}

\author{
Yi Lu, ${ }^{1,2, *}$ Muyang Chen, ${ }^{3}$ Zhan Bai, ${ }^{1,2, \dagger}$ Fei Gao, ${ }^{4}$ and Yu-xin Liu ${ }^{1,2,5, \$}$ \\ ${ }^{1}$ Department of Physics and State Key Laboratory of Nuclear Physics and Technology, \\ Peking University, Beijing 100871, China \\ ${ }^{2}$ Collaborative Innovation Center of Quantum Matter, Beijing 100871, China \\ ${ }^{3}$ Department of Physics, Hunan Normal University, Changsha 410081, China \\ ${ }^{4}$ Institut für Theoretische Physik, Universität Heidelberg, \\ Philosophenweg 16, 69120 Heidelberg, Germany \\ ${ }^{5}$ Center for High Energy Physics, Peking University, Beijing 100871, China
}

(Received 27 October 2015; revised 9 December 2021; accepted 3 January 2022; published 15 February 2022)

\begin{abstract}
By analyzing the calculated baryon number susceptibility ratios $\chi_{1}^{B} / \chi_{2}^{B}$ and $\chi_{3}^{B} / \chi_{1}^{B}$ in a two-flavor system via the Dyson-Schwinger equation approach of QCD, we determine the chemical freeze-out temperature and baryon chemical potential in cases of both thermodynamic limit and finite size. We calculate the centerof-mass energy dependence of the $\chi_{4}^{B} / \chi_{2}^{B}\left(\kappa \sigma^{2}\right)$ at the freeze-out line and find an excellent agreement with experimental data when taking into account the finite size effect. Our calculations indicate that the $\kappa \sigma^{2}$ exhibits a nonmonotonic behavior in the lower collision energy region. We also predict that the collision energy dependence of $\chi_{6}^{B} / \chi_{2}^{B}$ is nonmonotonic.
\end{abstract}

DOI: 10.1103/PhysRevD.105.034012

\section{INTRODUCTION}

Phase transitions of strong interaction matter have been explored for more than 40 years since the research may reveal the nature of the early Universe matter evolution [1-3]. The transitions include chiral phase transition (from dynamical chiral symmetry to dynamical chiral symmetry breaking) which generates more than $98 \%$ of the mass of visible matter and the confinement transition (hadronization) which binds the quarks and gluons to hadrons. They are driven by the temperature $(T)$ and the baryon density $\left(\rho_{B}\right)$ or chemical potential $\left(\mu_{B}\right)$. Since the strong interaction can be well described by quantum chromodynamics (QCD), the above mentioned phase transitions are usually referred to as QCD phase transitions. Moreover, many calculations (see, e.g., Refs. [2-19]) have shown that the chiral phase transition at low chemical potential is a crossover at physical quark mass. Theoretical calculations (see, e.g., Refs. [3,6,8-21]) also indicate that the chiral phase transition at high chemical potential is first order. Therefore, there would exist a critical end point (CEP) in the $T-\mu_{B}$ plane at which the first-order

\footnotetext{
*qwertylou@pku.edu.cn

'Present address: Institute of Theoretical Physics, Chinese Academy of Science, Beijing 100081, China.

Corresponding author. yxliu@pku.edu.cn

Published by the American Physical Society under the terms of the Creative Commons Attribution 4.0 International license. Further distribution of this work must maintain attribution to the author(s) and the published article's title, journal citation, and DOI. Funded by SCOAP ${ }^{3}$.
}

phase transition turns to crossover. The position of the CEP or even its existence becomes thus one of a most significant topic in both theories and experiments. Besides the efforts in theories, the Beam Energy Scan (BES) program at relativistic heavy ion collider (RHIC), the facility for antiproton and ion research (FAIR) at Gesellschaft für Schwerionenforschung (GSI), and the nuclotron-based ion collider facility (NICA) at Dubna in Russia all take the search of the CEP as their investigation focus (see, e.g., Refs. [22-24]), and some meaningful information has been provided by the RHIC experiments [25-28].

In experiments, one can measure only the states after the hadronization but not the phase transition directly, and thus, the chemical freeze-out line which is defined as the set of states ceasing the inelastic collision of the newly formed hadrons plays an essential role. Especially, as the chemical freeze-out line approaches the CEP, the nonmonotonic behavior of conserved charge fluctuations could be observed [9,29-34]. The freeze-out temperature and chemical potential have then been studied in a statistical hadronization model (SHM) [35-39], hadron resonance gas (HRG) model [40,41], lattice QCD simulations [42-45], and other models [46,47]. In fact, the matter system generated in the RHIC experiment has a finite size and cools in a finite time $[32,33,48,49]$. The finite size and finite time prevent the correlation length $\xi$ from diverging near the CEP and smoothen the fluctuations [48]. Model calculations have shown that the finite size influences both the phase diagram and the thermodynamical properties drastically [50-55], and the surface of the system may also play a role [56-61]. The effects of the finite size and the 
surface on the chemical freeze-out parameters will then complement the information for searching for the CEP in experiments. However, different models give contradictory results. It is therefore imperative to investigate the finite size and the surface effects on the chemical freeze-out parameters with sophisticated QCD approaches.

It has been known that Dyson-Schwinger equations (DSE), a nonperturbative approach of QCD [62-70], are successful in describing QCD phase transitions (see, e.g., Refs. [8-13,66,71-76]) and hadron properties (for recent reviews, see Refs. [66,67]). We then, in this paper, take the DSE approach to investigate the chemical freeze-out parameters with the finite size and surface effects taken into account. We calculate the baryon number susceptibilities in a two-light-flavor quark system. By comparing the obtained baryon number susceptibility ratios $\chi_{1}^{B} / \chi_{2}^{B}$ and $\chi_{3}^{B} / \chi_{1}^{B}$ with the experimental data of the net-proton distribution cumulant ratios $C_{1} / C_{2}$ and $C_{3} / C_{1}$ at different collision energies, we determine the freeze-out parameters. We observe that with the finite size and surface effects being included, the calculated collision energy dependence of the $\chi_{4}^{B} / \chi_{2}^{B}$ agrees with the experimental data excellently, and the calculated $\kappa \sigma^{2}$ shows a nonmonotonic behavior in the lower collision energy region. Moreover, we propose that a hyperorder cumulant ratio such as $\chi_{6}^{B} / \chi_{2}^{B}$ also shows a nonmonotonic dependence on the collision energy.

The remainder of this paper is organized as follows. In Sec. II, we describe briefly the Dyson-Schwinger equation approach and its relation to the chemical freeze-out parameters. In Sec. III, we calculate the freeze-out parameters by adopting the DSE approach as well as the experimental data. In Sec. IV, we give the phase diagram in the vicinity of CEP and reveal the effect of the finite size and surface. In Sec. V, we give a summary and discussion.

\section{THEORETICAL FRAMEWORK}

\section{A. Dyson-Schwinger equation approach}

The Dyson-Schwinger equations are an infinite number of coupled equations. In this paper, we focus on the DSE for quark propagator $S\left(\tilde{\omega}_{j}, \vec{p}\right)$. The corresponding equation is

$$
S^{-1}\left(\tilde{\omega}_{j}, \vec{p}\right)=Z_{2}\left(i \vec{\gamma} \cdot \vec{p}+i \gamma_{4} \tilde{\omega}_{j}\right)+Z_{4} m_{0}+Z_{1} \Sigma\left(\tilde{\omega}_{j}, \vec{p}\right),
$$

where $Z_{1}, Z_{2}$, and $Z_{4}$ are renormalization constants. $m_{0}$ is the current quark mass. $\tilde{\omega}_{j}=\omega_{j}+i \mu_{q}$, with $\mu_{q}$ being the quark chemical potential, and $\omega_{j}=(2 j+1) \pi T$, the Matsubara frequency for quarks. $\Sigma\left(\tilde{\omega}_{j}, \vec{p}\right)$ is the self-energy of quark, and reads as

$$
\begin{aligned}
\Sigma\left(\tilde{\omega}_{j}, \vec{p}\right)= & \frac{4}{3} T \sum_{l=-\infty}^{\infty} \int \frac{d^{3} \vec{q}}{(2 \pi)^{3}} g^{2} D_{\mu \nu}\left(\vec{k}, \Omega_{j l} ; T, \mu_{q}\right) \\
& \times \gamma_{\mu} S\left(\tilde{\omega}_{l}, \vec{q}\right) \Gamma_{\nu}\left(\vec{p}, \tilde{\omega}_{j}, \vec{q}, \tilde{\omega}_{l} ; T, \mu_{q}\right),
\end{aligned}
$$

where $D_{\mu \nu}$ is the dressed-gluon propagator, $\Gamma_{\nu}$ the dressed quark-gluon vertex, $\vec{k}=\vec{p}-\vec{q}$ the gluon momentum and $\Omega_{j l}=\omega_{j}-\omega_{l}$ the Matsubara frequency for gluon.

In principle, the gluon propagator $D_{\mu \nu}$ and the quarkgluon vertex $\Gamma_{\nu}$ should be solved by corresponding DSEs, which depend on higher-order correlation functions, and a truncation must be applied in order for numerical solution. In this paper, for the quark-gluon vertex, we adopt at first stage the rainbow approximation for the vertex $\Gamma_{\nu}\left(\vec{p}, \tilde{\omega}_{m}, \vec{q}, \tilde{\omega}_{l} ; T, \mu_{q}\right)=\gamma_{\nu}$.

The gluon propagator has the general form of

$g^{2} D_{\mu \nu}\left(\Omega_{n l}, \vec{k}\right)=P_{\mu \nu}^{T} D_{T}\left(\Omega_{n l}^{2}, \vec{k}^{2}\right)+P_{\mu \nu}^{L} D_{L}\left(\Omega_{n l}^{2}, \vec{k}^{2}\right)$,

where $P_{\mu \nu}^{T, L}$ are the transverse and longitudinal projection operators, respectively,

$$
\begin{aligned}
& P_{\mu \nu}^{T}=\left(1-\delta_{\mu 4}\right)\left(1-\delta_{\nu 4}\right)\left(\delta_{\mu \nu}-\frac{k_{\mu} k_{\nu}}{k^{2}}\right), \\
& P_{\mu \nu}^{L}=\left(\delta_{\mu \nu}-\frac{k_{\mu} k_{\nu}}{k^{2}}\right)-P_{\mu \nu}^{T},
\end{aligned}
$$

where $k=\left(\Omega_{n l}, \vec{k}\right) . D_{T}$ and $D_{L}$ are the effective interactions and can be represented using models. Note that the coupling constant $g$ and the renormalization constant $Z_{1}$ have been absorbed into the effective interaction.

In this paper, we adopt the infrared constant model (QC model) $[9,13,70]$, which reads as

$$
\mathcal{D}(s)=8 \pi^{2} \frac{D}{\omega^{4}} \mathrm{e}^{-s / \omega^{2}}+\alpha_{\mathrm{pQCD}}(s)
$$

where $D$ and $\omega$ are the parameters of the model. $\alpha_{\mathrm{pQCD}}$ is the ultraviolet perturbation term and reads as

$$
\alpha_{\mathrm{pQCD}}(s)=\frac{8 \pi^{2} \gamma_{m}}{\ln \left[\tau+\left(1+s / \Lambda_{\mathrm{QCD}}^{2}\right)^{2}\right]} \mathcal{F}(s),
$$

where $\mathcal{F}(s)=\left[1-\exp \left(-s / 4 m_{t}^{2}\right)\right], \tau=e^{2}-1, m_{t}=0.5 \mathrm{GeV}$, $\Lambda_{\mathrm{QCD}}=0.234 \mathrm{GeV}$, and $\gamma_{m}=12 /\left(33-N_{f}\right)$ with $N_{f}=4$.

The gluon screening mass $m_{g}$ is also considered in the longitudinal part of gluon model [13,77],

$$
\begin{aligned}
& D_{T}\left(\Omega_{n l}^{2}, \vec{k}^{2}\right)=\mathcal{D}\left(\Omega_{n l}^{2}+\vec{k}^{2}\right), \\
& D_{L}\left(\Omega_{n l}^{2}, \vec{k}^{2}\right)=\mathcal{D}\left(\Omega_{n l}^{2}+\vec{k}^{2}+m_{g}^{2}\right),
\end{aligned}
$$

whose value is determined by leading-order perturbative QCD [78],

$$
m_{g}^{2}=\frac{16}{5}\left(T^{2}+\frac{6}{5 \pi^{2}} \mu_{q}^{2}\right) .
$$

In order to fix the renormalization constants $Z_{2}$ and $Z_{4}$, we need to specify the renormalization condition. In this paper, the renormalization condition is 


$$
\left.S^{-1}\left(p^{2}\right)\right|_{\omega_{0}^{2}+\vec{p}^{2}=\zeta^{2}}=i\left(\vec{\gamma} \cdot \vec{p}+\gamma_{4} \tilde{\omega}_{0}\right)+\hat{m},
$$

where $\zeta$ is the renormalization point, and $\hat{m}$ is the renormalization-group invariant current-quark mass. We take parameters $\zeta=19 \mathrm{GeV}, \quad m_{0}=3.4 \mathrm{MeV}$, $D=1.024 \mathrm{GeV}^{2}$, and $\omega=0.5 \mathrm{GeV}$ as in Ref. [70] in our calculations. And the obtained renormalization-group invariant current-quark mass is $\hat{m}=6.0 \mathrm{MeV}$.

The quark propagator can be decomposed according to its Lorentz structure. At finite temperature, the decomposition is

$$
\begin{aligned}
S\left(\tilde{\omega}_{j}, \vec{p}\right)^{-1}= & i \vec{\gamma} \cdot \vec{p} A\left(\tilde{\omega}_{j}^{2}, \vec{p}^{2}\right) \\
& +i \gamma_{4} \tilde{\omega}_{j} C\left(\tilde{\omega}_{j}^{2}, \vec{p}^{2}\right)+B\left(\tilde{\omega}_{j}^{2}, \vec{p}^{2}\right) .
\end{aligned}
$$

There should be, in principle, a fourth term in this decomposition. However, its contribution to order parameter is extremely small and is usually omitted in practical calculations $[63,79]$.

The mass function of the quark propagator can then be defined as

$$
M\left(\tilde{\omega}_{j}^{2}, \vec{p}^{2}\right)=B\left(\tilde{\omega}_{j}^{2}, \vec{p}^{2}\right) / A\left(\tilde{\omega}_{j}^{2}, \vec{p}^{2}\right) .
$$

In a vacuum, the physical solution to the quark DSE has a nonzero mass function even if the current quark mass is zero. At high temperature, the mass function gradually approaches the current quark mass. Therefore, the mass function at zero momentum, $M\left(\tilde{\omega}_{0}^{2}, 0\right)$, is often used as the order parameter of the QCD crossover.

\section{B. Number density and susceptibilities}

Experimental observations indicate that the yields of pions and protons are much larger than that of kaons [38]; we can then simplify the matter generated in RHIC experiments as that including mainly two-light-flavor quarks. In the system of $u$ and $d$ quarks, baryon number density $n_{B}$ and electric charge density $n_{Q}$ can be fixed with quark number density $n_{u, d}$ as

$$
n_{B}=\frac{1}{3} n_{u}+\frac{1}{3} n_{d}, \quad n_{Q}=\frac{2}{3} n_{u}-\frac{1}{3} n_{d} .
$$

From Eq. (12), we notice that the $u$ and $d$ quarks are in exact isospin symmetry, if only the baryon number is considered. In this sense, both the $u$ quark and $d$ quark hold the same quark chemical potential $\mu_{q}=\mu_{B} / 3$ and the quark number density $n_{q}=3 n_{B}$.

In view of statistical physics, the quark number density can be determined as

$$
\begin{aligned}
n_{q}\left(\mu_{q}, T\right) & =2 N_{c} N_{f} Z_{2} \int \frac{d^{3} \vec{p}}{(2 \pi)^{3}} f_{1}\left(|\vec{p}| ; \mu_{q}, T\right), \\
f_{1}\left(|\vec{p}| ; \mu_{q}, T\right) & =\frac{T}{2} \sum_{j=-\infty}^{\infty} \operatorname{tr}_{D}\left[-\gamma_{4} S\left(\tilde{\omega}_{j}, \vec{p}\right)\right],
\end{aligned}
$$

where $Z_{2}$ is the quark wave function renormalization constant, $N_{c}=3$ the color number, and $N_{f}=2$ the flavor number. Notice that the flavor number here represents the flavor degeneracy and is different from the one we used in the ultraviolet perturbation term in the gluon model.

In Eq. (14), the summation runs over an infinite number of Matsubara frequencies. In practice, we can only carry out the calculation with a finite number of Matsubara frequencies, and the summation must have a cutoff, $N$. However, the convergence of summation Eq. (14) is slow, especially at low temperature. On the other hand, we observe that the scalar functions $A, B$, and $C$ defined in Eq. (10) converge quickly to the free-quark-propagator scalar functions $A=C=1, B=m_{0}$, as the Matsubara frequency grows large. For a free propagator, the distribution function is

$$
\begin{aligned}
f^{\text {free }}\left(|\vec{p}| ; \mu_{q}, T\right) & =\frac{T}{2} \sum_{j=-\infty}^{\infty} \operatorname{tr}_{D}\left[-\gamma_{4} S^{\mathrm{free}}\left(\tilde{\omega}_{j}, \vec{p}\right)\right] \\
& =\frac{1}{\mathrm{e}^{\left(E-\mu_{q}\right) / T}+1}-\frac{1}{\mathrm{e}^{\left(E+\mu_{q}\right) / T}+1},
\end{aligned}
$$

where $E=\sqrt{\vec{p}^{2}+m_{0}^{2}}$.

Therefore, the contribution of missing Matsubara frequencies that exceed the cutoff $N$ in Eq. (14) can be approximated using the distribution function of free propagator,

$$
\begin{aligned}
f_{1}= & \frac{T}{2} \sum_{j=-N}^{N} \operatorname{tr}_{D}\left[-\gamma_{4} S\left(\tilde{\omega}_{j}, \vec{p}\right)\right] \\
& +f^{\text {free }}-\frac{T}{2} \sum_{j=-N}^{N} \operatorname{tr}_{D}\left[-\gamma_{4} S^{\text {free }}\left(\tilde{\omega}_{j}, \vec{p}\right)\right] .
\end{aligned}
$$

Using this technique, the calculated quark number density has better convergence at moderate and high temperature, whose chemical potential dependence is shown in Fig. 1.

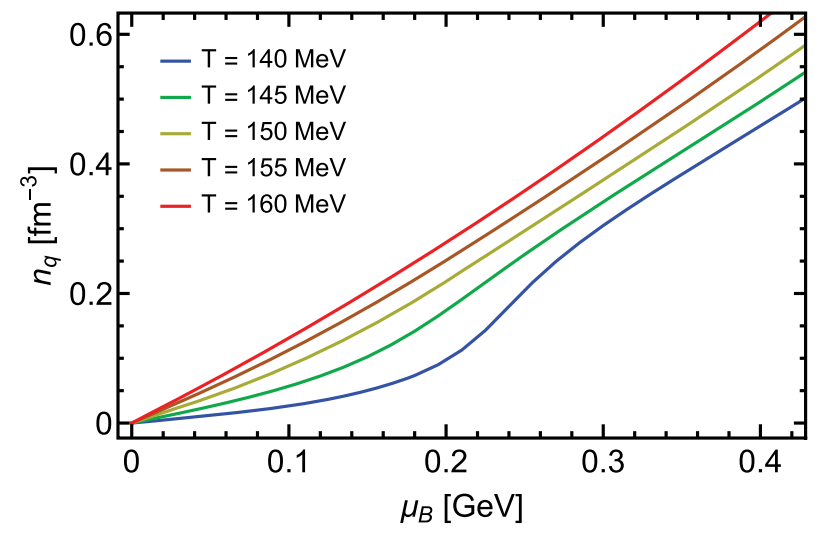

FIG. 1. Calculated baryon chemical potential dependence of the quark number density at some values of given temperature, in the case of a thermodynamical limit using Eq. (16). 
After calculating the quark number density, we can calculate the susceptibility by taking derivatives. The $k$ th order baryon number density susceptibility (fluctuation) is obtained as

$$
\begin{aligned}
& \chi_{1}^{B}=n_{B}, \\
& \chi_{k}^{B}=\frac{1}{\beta^{(k-1)}} \frac{\partial^{(k-1)} n_{B}}{\partial^{(k-1)} \mu_{B}},
\end{aligned}
$$

where $\beta=1 / T$ and $k=2,3,4, \ldots$. The susceptibilities are related to the moments of the multiplicity distributions of the corresponding conserved charges as

$$
\begin{aligned}
& \frac{\chi_{1}}{\chi_{2}}=M / \sigma^{2}, \quad \frac{\chi_{3}}{\chi_{1}}=S \sigma^{3} / M, \\
& \frac{\chi_{3}}{\chi_{2}}=S \sigma, \quad \frac{\chi_{4}}{\chi_{2}}=\kappa \sigma^{2},
\end{aligned}
$$

where $M, \sigma^{2}, S$, and $\kappa$ are the mean, the variance, the skewness, and the kurtosis of the multiplicity distribution, respectively. By comparing the theoretical net-baryon number fluctuations in terms of temperature and chemical potential with the experimental data, one can determine the freeze-out parameters [45].

\section{Finite size and surface effect}

The system created in RHIC exists in finite size, rather than thermodynamical limit. To determine the freeze-out parameters in the experiment, one has to take the finite size and the surface effects into account.

Consider a system with a cubic boundary of size $L$. This corresponds to the antiperiodic condition on the quark field, whose spatial momentum becomes $p_{j}=(2 j+1) \pi / L$, and one should deal with discrete momentum values of integer $j$ [80]. However, for the system with a spherical or almondshaped boundary, which is more suitable to describe the actual physics case of a quark-gluon plasma (QGP) fireball, the discrete momentum approach is complicated. In this work, the finite size effect is studied with the picture of a quark liquid droplet, and the theory is constructed in continuous momentum space. In the quark DSE, the inhomogeneous effects from the surface and curvature are neglected for simplicity. The finite volume effect of the system with size $L$ can then be roughly incorporated by an infrared momentum cutoff $|p|_{\min }=\pi / L[53,54]$ in the quark DSE Eqs. (1) and (2). It is remarkable that such an $L$ is not the same as the size of the fireball but an effective scale that the ingredients of the quark matter can interact with each other.

In the calculation of quark number density, the surface and curvature effects are included through the multiple reflection expansion (MRE) approximation. In the MRE approximation, the thermodynamical quantities of the droplet composed of quarks can be derived from a density of states in the form [56-58,81,82]

$$
\frac{d N}{d p}=6\left[\frac{p^{2} V}{2 \pi^{2}}+f_{s}\left(\frac{p}{M}\right) p S+f_{c}\left(\frac{p}{M}\right) C+\cdots\right],
$$

where $V$ is the volume of the droplet, and $S$ and $C$ are the area and the extrinsic curvature of the surface of the droplet, respectively.

For the spherical boundary condition, $V=4 \pi L^{3} / 3$, $S=4 \pi L^{2}$, and $C=8 \pi L$, and the structure functions $f_{s}$ and $f_{c}$ are given explicitly as $[52,81]$

$$
\begin{aligned}
& f_{s}\left(\frac{p}{M}\right)=-\frac{1}{8 \pi}\left(1-\frac{2}{\pi} \arctan \left(\frac{p}{M}\right)\right), \\
& f_{c}\left(\frac{p}{M}\right)=\frac{1}{12 \pi^{2}}\left[1-\frac{3 p}{2 M}\left(\frac{\pi}{2}-\arctan \left(\frac{p}{M}\right)\right)\right],
\end{aligned}
$$

with $p$ being the momentum, and the constituent quark mass $M=\operatorname{Re} M\left(\tilde{\omega}_{0}^{2}, 0\right)$ is defined by the mass function in Eq. (11).
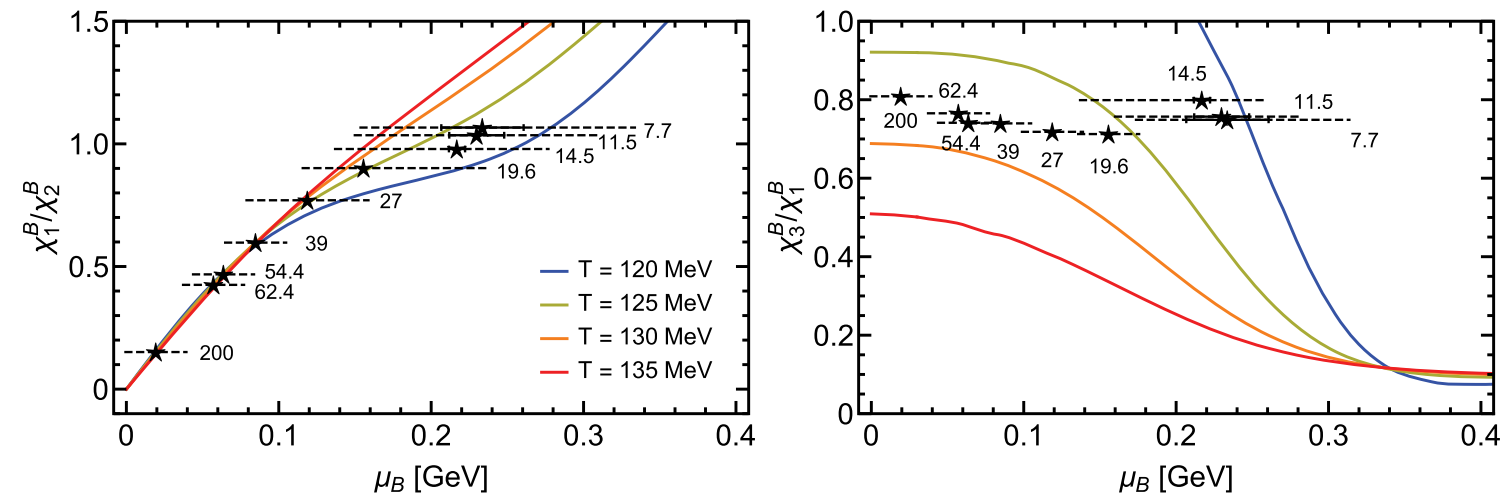

FIG. 2. Calculated baryon chemical potential dependence of the fluctuation ratios $\chi_{1}^{B} / \chi_{2}^{B}$ (left panel) and $\chi_{3}^{B} / \chi_{1}^{B}$ (right panel) of the system with $L=2.2 \mathrm{fm}$ at several values of temperature near the $T_{c}$. The dashed horizontal lines stand for the experimental values of the efficiency-corrected $C_{1} / C_{2}=M / \sigma^{2}$ and $C_{3} / C_{1}=S \sigma^{3} / M$ of net-proton multiplicity distributions in the central collisions at $\sqrt{S_{N N}}=200,62.4,54.4,39,27,19.6,14.5,11.5,7.7 \mathrm{GeV}$ given in Ref. [28]. The stars label our assigned freeze-out points. 
TABLE I. Calculated freeze-out points $\left(\mu_{B}^{f}, T^{f}\right)$ in the case of different values of $L\left(T^{f}\right.$ and $\mu_{B}^{f}$ are in unit MeV and $\sqrt{S_{N N}}$ in GeV).

\begin{tabular}{|c|c|c|c|c|c|c|c|c|c|c|}
\hline \multirow[b]{2}{*}{$\sqrt{S_{N N}}$} & \multicolumn{2}{|c|}{$L=$ infinity } & \multicolumn{2}{|c|}{$L=3.5 \mathrm{fm}$} & \multicolumn{2}{|c|}{$L=2.8 \mathrm{fm}$} & \multicolumn{2}{|c|}{$L=2.5 \mathrm{fm}$} & \multicolumn{2}{|c|}{$L=2.2 \mathrm{fm}$} \\
\hline & $\mu_{B}^{f}$ & $T^{f}$ & $\mu_{B}^{f}$ & $T^{f}$ & $\mu_{B}^{f}$ & $T^{f}$ & $\mu_{B}^{f}$ & $T^{f}$ & $\mu_{B}^{f}$ & $T^{f}$ \\
\hline 200 & 23.7 & 155.5 & 22.8 & 150.0 & 21.9 & 144.1 & 21.1 & 138.8 & 19.4 & 127.5 \\
\hline 62.4 & 69.9 & 155.1 & 67.1 & 150.0 & 64.4 & 144.0 & 62.1 & 138.9 & 57.2 & 128.0 \\
\hline 54.4 & 77.2 & 155.1 & 74.6 & 150.0 & 70.2 & 144.3 & 69.1 & 139.1 & 63.8 & 128.4 \\
\hline 39 & 103.3 & 154.2 & 99.5 & 149.2 & 95.5 & 143.5 & 92.0 & 138.5 & 84.8 & 128.1 \\
\hline 27 & 154.7 & 150.7 & 143.0 & 147.1 & 135.4 & 141.8 & 129.6 & 137.3 & 118.8 & 127.5 \\
\hline \multirow[t]{2}{*}{19.6} & 189.1 & 148.0 & 191.7 & 143.1 & 195.2 & 137.5 & 178.7 & 134.1 & 155.7 & 126.0 \\
\hline & \pm 15.7 & \pm 1.9 & \pm 10.9 & \pm 1.2 & \pm 2.5 & \pm 0.2 & & & & \\
\hline \multirow[t]{2}{*}{14.5} & $\ldots$ & $\ldots$ & $\ldots$ & $\ldots$ & 241.5 & 132.0 & 203.3 & 131.6 & 217.0 & 121.9 \\
\hline & & & & & \pm 21.1 & \pm 2.7 & \pm 23.8 & \pm 2.2 & \pm 5.3 & \pm 0.4 \\
\hline \multirow[t]{2}{*}{11.5} & $\ldots$ & $\ldots$ & $\ldots$ & $\ldots$ & $\ldots$ & $\ldots$ & 255.0 & 127.7 & 229.9 & 121.4 \\
\hline & & & & & & & \pm 34.3 & \pm 2.3 & \pm 18.0 & \pm 1.4 \\
\hline \multirow[t]{2}{*}{7.7} & $\ldots$ & $\ldots$ & $\ldots$ & $\ldots$ & $\ldots$ & $\ldots$ & 257.4 & 127.5 & 233.7 & 121.2 \\
\hline & & & & & & & \pm 39.9 & \pm 2.9 & \pm 27.0 & \pm 2.1 \\
\hline
\end{tabular}

The modified density of state is then $[52,82]$

$$
\rho_{\mathrm{MRE}}(p, M, L)=1+\frac{6 \pi^{2}}{p L} f_{s}+\frac{12 \pi^{2}}{(p L)^{2}} f_{c},
$$

and the momentum integration in Eq. (13) is converted as

$$
\int_{0}^{\infty} \frac{\vec{p}^{2} \mathrm{~d} p}{2 \pi^{2}} \rightarrow \int_{|p|_{\min }}^{\Lambda} \rho_{\mathrm{MRE}} \frac{\vec{p}^{2} \mathrm{~d} p}{2 \pi^{2}} .
$$

Notice that the MRE density of state becomes negative at very low momentum; this is because it cannot be applied in the $p \lesssim|p|_{\min }$ region due to the finite volume restriction. We then choose $|p|_{\min }$ as the lower limit of integration, Eq. (22).

\section{FREEZE-OUT PARAMETERS}

We have carried out calculations with $L=\infty$ (thermodynamical limit) and various finite values of $L$. The calculations manifest that the fluctuations (skewness, kurtosis, etc.) in the $T-\mu_{B}$ plane behave qualitatively the same as those given in Ref. [9], respectively. The obtained $\mu_{B}$ dependence of the baryon number susceptibility ratios $\chi_{1}^{B} / \chi_{2}^{B}$ and $\chi_{3}^{B} / \chi_{1}^{B}$ in the case of $L=2.2 \mathrm{fm}$ at several values of temperature are shown in Fig. 2. It is evident that our results agree with the lattice QCD results [45] qualitatively very well. In order to extract the freeze-out parameters, we plot the experimental values of the cumulant ratios $C_{1} / C_{2}=M / \sigma^{2}$ and $C_{3} / C_{1}=S \sigma^{3} / M$ of netproton multiplicity distributions in central collisions [28] as horizontal lines.

By fitting our calculated $\chi_{1}^{B} / \chi_{2}^{B}$ and $\chi_{3}^{B} / \chi_{1}^{B}$ values in terms of $T$ and $\mu_{B}$ with the experimental data, we get the freeze-out parameters $\left(\mu_{B}^{f}, T^{f}\right)$. The obtained results for $L=\infty, 3.5,2.8,2.5$, and $2.2 \mathrm{fm}$ are listed in Table I.
It appears that our theoretical results in the thermodynamical limit $(L=\infty)$ do not fit the experimental values well at low collision energy, whereas the deviations are smaller if the finite size parameter $L$ changes.

We illustrate the presently calculated relation between the baryon chemical potential $\mu_{B}^{f}$ and the center-of-mass energy of the collision, $\sqrt{S_{N N}}$, and the comparison with those given in lattice QCD simulations (e.g., Ref. [45]) and model calculations (e.g., Refs. [36,41]) in Fig. 3. We see from Fig. 3 that our freeze-out baryon chemical potential in the case of $L=\infty$ and when $L=2.2 \mathrm{fm}$ match the lattice QCD result and model calculation results well in the region $\mu_{B}<100 \mathrm{MeV}$, while those in the case of $L=2.2 \mathrm{fm}$ deviate from previous results in the $\mu_{B}>100 \mathrm{MeV}$ range. With the obtained freeze-out points, the freeze-out conditions are fitted as

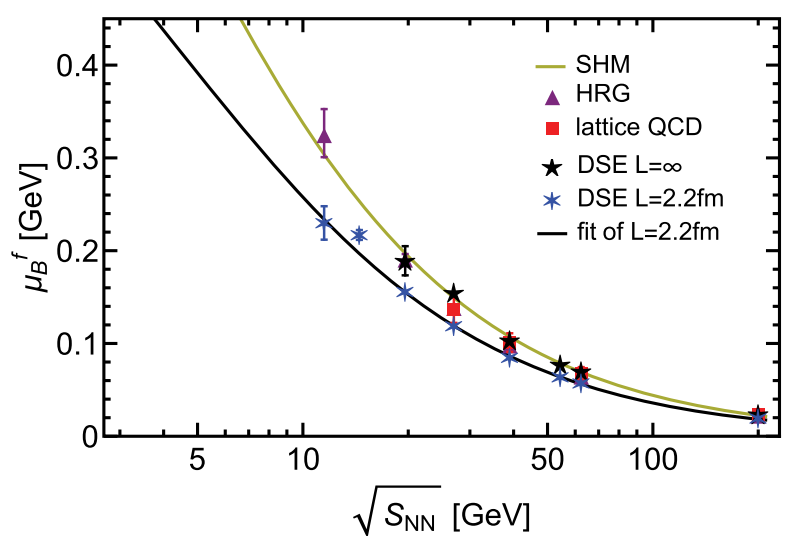

FIG. 3. Comparison of presently obtained $\sqrt{S_{N N}}$ dependence of the baryon chemical potential in cases of $L=\infty$ and $L=2.2 \mathrm{fm}$ with those given in the lattice QCD simulation [45], HRG model [41], and parameterized one in the SHM model [36]. 
TABLE II. Fitted freeze-out parameters $c, d$ in Eq. (23) and $T^{0}, a, b$ in Eq. (24), in case of different finite size parameter $L$ ( $c$ and $T^{0}$ are in unit $\mathrm{MeV}$ and $d$ in $\mathrm{GeV}^{-1}$ ).

\begin{tabular}{lrrrcc}
\hline \hline$L$ & $c$ & $d$ & $T^{0}$ & $a$ & $b$ \\
\hline Infinity & 1642.8 & 0.373 & 155.7 & 0.0162 & 0.0166 \\
$3.5 \mathrm{fm}$ & 1236.1 & 0.285 & 150.3 & 0.0130 & 0.0103 \\
$2.8 \mathrm{fm}$ & 1066.9 & 0.256 & 144.6 & 0.0184 & 0.0043 \\
$2.5 \mathrm{fm}$ & 840.9 & 0.205 & 139.2 & 0.0137 & 0.0051 \\
$2.2 \mathrm{fm}$ & 818.4 & 0.218 & 128.4 & 0.0106 & 0.0022 \\
\hline \hline
\end{tabular}

$$
\begin{aligned}
\mu_{B}^{f} & =\frac{c}{1+d \sqrt{S_{N N}}}, \\
T^{f} & =T^{0}\left[1-a\left(\frac{\mu_{B}^{f}}{T^{0}}\right)^{2}-b\left(\frac{\mu_{B}^{f}}{T^{0}}\right)^{4}\right] .
\end{aligned}
$$

Only the freeze-out points with small deviation between theory and experiment in Table I are used for fitting the freeze-out conditions, and the obtained best-fitted parameters are listed in Table II. The fitted $\mu_{B}^{f}\left(\sqrt{S_{N N}}\right)$ curve is also displayed in Fig. 3.

With the parametrization, one can predict the freeze-out parameters $\left(\mu_{B}^{f}, T^{f}\right)$ of the system generated in any collision energy. For example, with $L=2.2 \mathrm{fm}, \sqrt{S_{N N}}=5.8$, 7.7, 11.5 , and $14.5 \mathrm{GeV}$ correspond to $\left(\mu_{B}^{f}, T^{f}\right)=$ (361.7, 99.6), (305.8, 111.5), (233.6, 120.8), and (196.9, 123.6) $\mathrm{MeV}$, respectively.

\section{PHASE DIAGRAM AND FURTHER PREDICTION}

With the quark propagator obtained by solving the DSE, we can get the temperature and chemical potential dependence of the quark condensate and the quark dynamical mass, which are commonly regarded as appropriate order parameters of chiral phase transition. Taking the chiral susceptibility criterion $[8,77]$, we determine the upper and lower boundaries of the chiral phase crossover region by the full width at half maxima of the susceptibility. The chiral susceptibility is defined as

$$
\chi_{T}=-\partial M\left(\tilde{\omega}_{0}^{2}, 0\right) / \partial T,
$$

where the constituent quark mass is defined as the real part of $M\left(\tilde{\omega}_{0}^{2}, 0\right)$ in Eq. (11), the same as in Sec. IIC. The obtained crossover regions in the cases of $L=\infty, 2.5$, and $2.2 \mathrm{fm}$ are shown as the shadowed regions in Fig. 4.

In the low temperature and high chemical potential region, two separate solutions of the quark DSE are found simultaneously, namely, the Nambu and the Wigner solutions, respectively. This implies that even with a finite system size, there exists a first-order phase transition; hence, there is still a CEP in the $(T, \mu)$ plane.

With the chiral susceptibility criterion $[8,77]$ or the fluctuation criterion [9], we determine the boundaries of the first-order transition region and the location of the CEP. The obtained results in the two cases are displayed in Fig. 4.
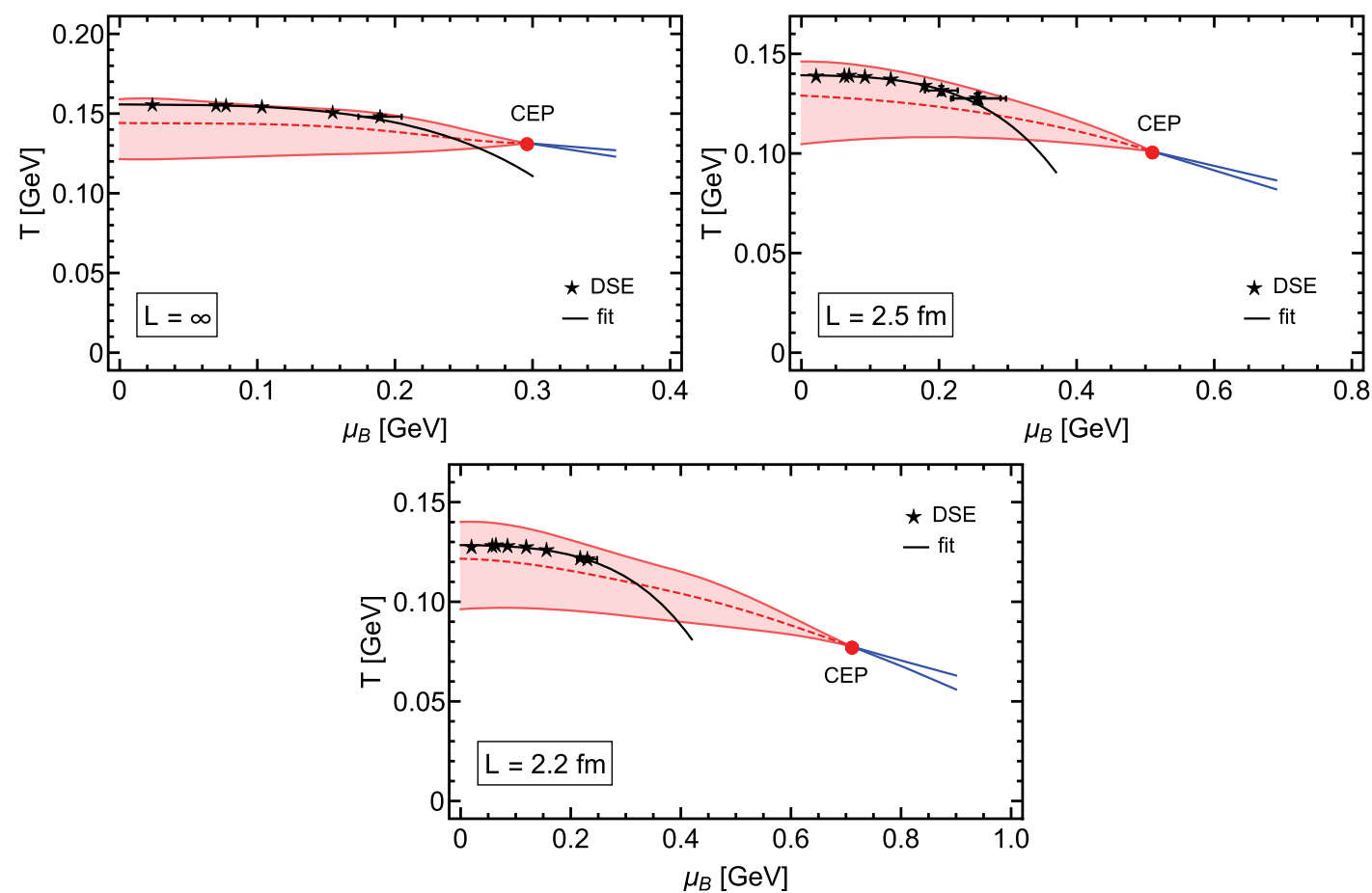

FIG. 4. Calculated QCD phase diagram in case of $L=\infty, 2.5 \mathrm{fm}$ and $2.2 \mathrm{fm}$. The red-dashed curves are the phase boundaries defined with the maximum of chiral susceptibility, the red-colored areas are the obtained crossover regions, and the black-solid curves are the fitted freeze-out lines. The blue-solid angles are the co-exist region of first-order phase transition. 
We illustrate also the presently obtained chemical freezeout lines in these cases in Fig. 4. The figure manifests that the chemical freeze-out happens in the obtained chiral crossover region. Quantitatively, the freeze-out temperature $T^{f}=155.7 \mathrm{MeV}$ is higher than the $T_{c}=144.0 \mathrm{MeV}$ in the thermodynamical limit (i.e., with $L=\infty$ ) at $\mu_{B}=0$, but the deviation is smaller in the case of $L=2.2 \mathrm{fm}$ where $T^{f}=128.4 \mathrm{MeV}$ and $T_{c}=121.6 \mathrm{MeV}$ at $\mu_{B}=0$. We also notice that the finite size effect shifts the location of the CEP to higher baryon chemical potential and lower temperature drastically; in the cases of $L=\infty, 3.5,2.8$, 2.5 , and $2.2 \mathrm{fm}, \quad\left(\mu_{B}^{\mathrm{CEP}}, T^{\mathrm{CEP}}\right)=(296,132),(360,121)$, $(426,111),(510,101),(711,77.5) \mathrm{MeV}$, respectively. This system-size dependence of the location of CEP is qualitatively consistent with other theoretical studies $[20,21,50,53,61,80,83]$. Since the BES program at RHIC has not shown any clear signal of the CEP in Au-Au collisions for collision energy down to $\sqrt{S_{N N}}=7.7 \mathrm{GeV}$, which implies that $\mu_{B}^{\mathrm{CEP}} \gtrsim 400 \mathrm{MeV}$ according to Refs. [38,39], we infer that the finite size parameter satisfies $L \lesssim 2.8 \mathrm{fm}$ for the QGP system produced in the $\mathrm{Au}-\mathrm{Au}$ collision. We emphasize again that this $L$ is a semiquantitative measure of the radius of fireball.

It is known that the $\kappa \sigma^{2}=\chi_{4} / \chi_{2}$ is a direct observable in the experiment and may demonstrate the property of the states around the CEP well. We calculate $\chi_{4}^{B} / \chi_{2}^{B}$ in the $T-\mu_{B}$ plane and pick out the value along the freeze-out line to get the $\sqrt{S_{N N}}$ dependence of $\chi_{4}^{B} / \chi_{2}^{B}$. The obtained results in the case of a thermodynamical limit and finite size of $L=2.8,2.5$, and $2.2 \mathrm{fm}$ are depicted in Fig. 5. In the case of $L=2.2 \mathrm{fm}$, the lowest collision energy calculated is

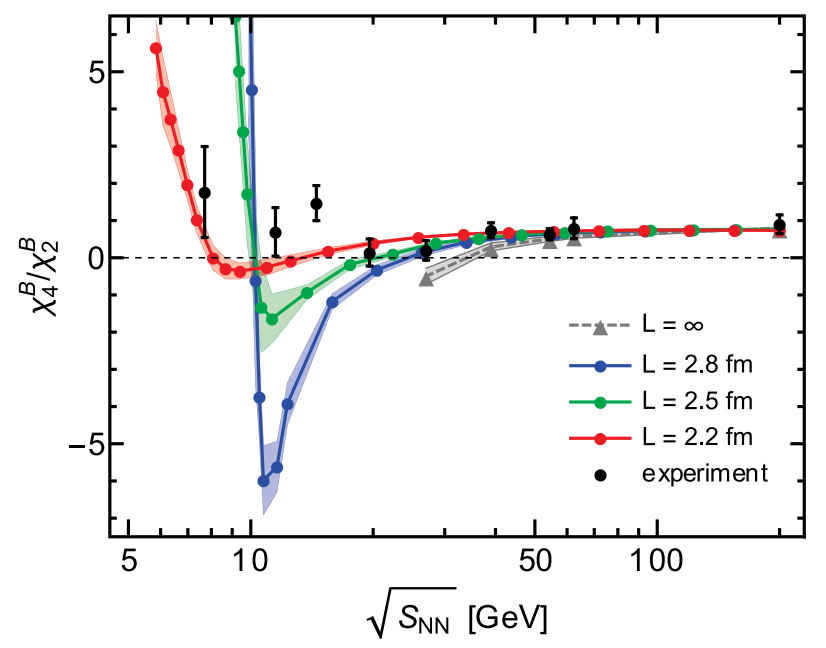

FIG. 5. Calculated collision energy $\sqrt{S_{N N}}$ dependence of $\kappa \sigma^{2}=\chi_{4}^{B} / \chi_{2}^{B}$ at the freeze-out line. The black circles are the experimental values [28]. The gray triangles stand for our results in case of infinite volume. The blue, green, and red points denote our results in the case of $L=2.8,2.5$, and $2.2 \mathrm{fm}$, respectively. The shadowed regions display the numerical uncertainties.

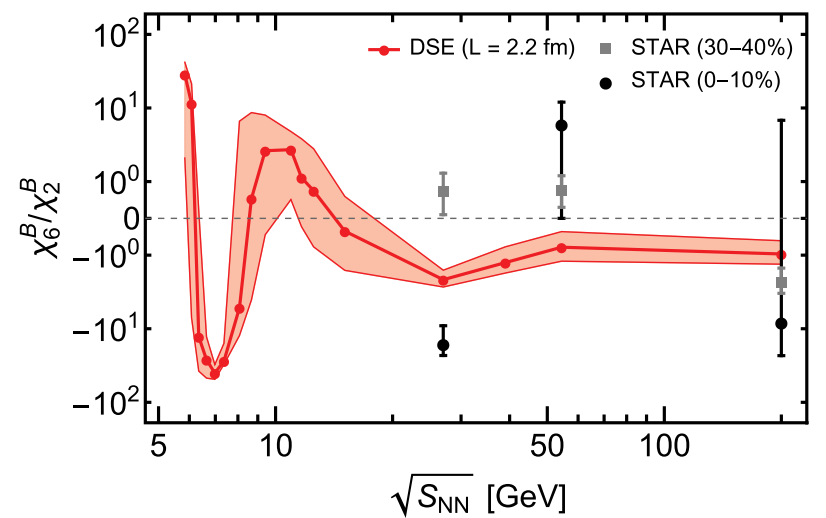

FIG. 6. Calculated collision energy $\sqrt{S_{N N}}$ dependence of $\chi_{6}^{B} / \chi_{2}^{B}$ (in logarithmic scale) at the freeze-out line. The black circles and gray squares are the experimental values [84] for $0-10 \%$ and $30-40 \%$ centralities, respectively, and the red points denote our results in the case of $2.2 \mathrm{fm}$. The shadowed regions display the numerical uncertainties.

$5.8 \mathrm{GeV}$. It is apparent that, without considering the finite size effect, our calculated $\chi_{4}^{B} / \chi_{2}^{B}$ decreases more rapidly than the experimental data as the $\sqrt{S_{N N}}$ descends. With the finite size effect being taken into account, we can reproduce the experimental data excellently. In the lower collision energy region, the $\kappa \sigma^{2}$ exhibits a nonmonotonic behavior, whose minimum is reached at $\sqrt{S_{N N}} \approx 10 \mathrm{GeV}$, and then increases drastically as $\sqrt{S_{N N}}$ further decreases.

Since the calculated kurtosis fit the experimental data best in the case of $L=2.2 \mathrm{fm}$, we further calculate the $\sqrt{S_{N N}}$ dependence of $\chi_{6}^{B} / \chi_{2}^{B}$ from our fitted freeze-out line under this finite size parameter. The obtained result is illustrated in Fig. 6. This shows that the numerical uncertainties are much larger than the results of $\kappa \sigma^{2}$.

At $\sqrt{S_{N N}}=200 \mathrm{GeV}$, we obtain a negative value of $\chi_{6}^{B} / \chi_{2}^{B}=-0.97 \pm 0.36$, which is qualitatively consistent with the experimental results [84-86] and lattice calculation [87]. For $\sqrt{S_{N N}} \gtrsim 10 \mathrm{GeV}$, our result shows a nonmonotonic behavior with a shallow minimum, in agreement with the functional renormalization group calculation [88]. We also show that $\chi_{6}^{B} / \chi_{2}^{B}$ may have a large maximum along with a sharp and deep minimum when $\sqrt{S_{N N}} \lesssim 10 \mathrm{GeV}$. As a result, we predict a complex nonmonotonic behavior of $\chi_{6}^{B} / \chi_{2}^{B}$ as a function of collision energy.

\section{SUMMARY}

In summary, we have calculated in this work the baryon number susceptibilities in a two-flavor quark system via the DSE approach of QCD in the case of not only the thermodynamic limit but also the finite size. By comparing the calculated ratios $\chi_{1}^{B} / \chi_{2}^{B}$ and $\chi_{3}^{B} / \chi_{1}^{B}$ with the experimental data of the net-proton multiplicity distribution in BES at RHIC, we obtained the temperature and the baryon 
chemical potential at the chemical freeze-out states. We calculated also the collision energy dependence of the $\kappa \sigma^{2}$ at the freeze-out line and observed an excellent agreement with experimental data when taking into account the finite size effect. It shows that the finite size effect is significant in studying the QCD phase transitions with RHICs. The obtained collision energy $\sqrt{S_{N N}}$ dependence of the $\kappa \sigma^{2}$ exhibits a nonmonotonic behavior in a lower collision energy region. We also predict that the collision energy dependence of hyperorder cumulant ratios such as $\chi_{6}^{B} / \chi_{2}^{B}$ may also be nonmonotonic.

\section{ACKNOWLEDGMENTS}

The work was supported by the National Natural Science Foundation of China under Grants No. 11175004 and No. 11435001 and the National Key Basic Research Program of China under Grant No. 2015CB856900.
[1] P. Braun-Munzinger and J. Wambach, Rev. Mod. Phys. 81, 1031 (2009).

[2] O. Philipsen, Prog. Part. Nucl. Phys. 70, 55 (2013).

[3] S. Gupta, X. Luo, B. Mohanty, H. G. Ritter, and N. Xu, Science 332, 1525 (2011).

[4] Y. Aoki, G. Endrődi, Z. Fodor, S. D. Katz, and K. K. Szabó, Nature (London) 443, 675 (2006).

[5] Y. Aoki, S. Borsányi, S. Dürr, Z. Fodor, S. D. Katz, S. Krieg, and K. Szabo, J. High Energy Phys. 06 (2009) 088.

[6] S. Ejiri, Phys. Rev. D 78, 074507 (2008).

[7] A. Li, A. Alexandru, and K.-F. Liu, Phys. Rev. D 84, 071503(R) (2011).

[8] S. X. Qin, L. Chang, H. Chen, Y. X. Liu, and C. D. Roberts, Phys. Rev. Lett. 106, 172301 (2011).

[9] X. Y. Xin, S. X. Qin, and Y. X. Liu, Phys. Rev. D 90, 076006 (2014).

[10] C. S. Fischer, J. Luecker, and J. A. Mueller, Phys. Lett. B 702, 438 (2011).

[11] C. S. Fischer and J. Luecker, Phys. Lett. B 718, 1036 (2013).

[12] C. S. Fischer, J. Luecker, and C. A. Welzbacher, Phys. Rev. D 90, 034022 (2014).

[13] F. Gao, J. Chen, Y. X. Liu, S. X. Qin, C. D. Roberts, and S. M. Schmidt, Phys. Rev. D 93, 094019 (2016).

[14] C. Ratti, M. A. Thaler, and W. Weise, Phys. Rev. D 73, 014019 (2006).

[15] B.-J. Schaefer, J. M. Pawlowski, and J. Wambach, Phys. Rev. D 76, 074023 (2007).

[16] W. J. Fu, Z. Zhang, and Y. X. Liu, Phys. Rev. D 77, 014006 (2008).

[17] K. Fukushima, Phys. Rev. D 77, 114028 (2008).

[18] L. J. Jiang, X. Y. Xin, K. L. Wang, S. X. Qin, and Y. X. Liu, Phys. Rev. D 88, 016008 (2013).

[19] X. Y. Xin, S. X. Qin, and Y. X. Liu, Phys. Rev. D 89, 094012 (2014).

[20] F. Gao and J. M. Pawlowski, Phys. Rev. D 102, 034027 (2020).

[21] W. J. Fu, J. M. Pawlowski, and F. Rennecke, Phys. Rev. D 101, 054032 (2020).

[22] G. L. Melkumov et al. (NA49 Collaboration), Nucl. Phys. B, Proc. Suppl. 219-220, 102 (2011).

[23] G. L. Melkumov et al. (NA49 Collaboration), Phys. At. Nucl. 75, 556 (2012).

[24] G. Odyniec, Phys. At. Nucl. 75, 602 (2012).
[25] L. Adamczyk et al. (STAR Collaboration), Phys. Rev. Lett. 112, 032302 (2014).

[26] L. Adamczyk et al. (STAR Collaboration), Phys. Rev. Lett. 113, 092301 (2014).

[27] X. Luo, Proc. Sci., CPOD2014 (2015) 019 [arXiv: 1503.02558].

[28] J. Adam et al. (STAR Collaboration), Phys. Rev. Lett. 126, 092301 (2021).

[29] M. Stephanov, K. Rajagopal, and E. Shuryak, Phys. Rev. Lett. 81, 4816 (1998).

[30] M. A. Stephanov, Phys. Rev. Lett. 102, 032301 (2009).

[31] M. A. Stephanov, Phys. Rev. Lett. 107, 052301 (2011).

[32] M. Stephanov, K. Rajagopal, and E. Shuryak, Phys. Rev. D 60, 114028 (1999).

[33] M. A. Stephanov, J. Phys. G 38, 124147 (2011).

[34] Y. Hatta and M. A. Stephanov, Phys. Rev. Lett. 91, 102003 (2003).

[35] F. Becattini, J. Manninen, and M. Gaździcki, Phys. Rev. C 73, 044905 (2006).

[36] A. Andronic, P. Braun-Munzinger, and J. Stachel, Nucl. Phys. A772, 167 (2006).

[37] A. Andronic, P. Braun-Munzinger, and J. Stachel, Phys. Lett. B 673, 142 (2009).

[38] S. Das (STAR Collaboration). J. Phys. Conf. Ser. 509, 012066 (2014).

[39] S. Das, EPJ Web Conf. 90, 10003 (2015).

[40] F. Karsch and K. Redlich, Phys. Lett. B 695, 136 (2011).

[41] P. Alba, W. Alberico, R. Bellwied, M. Bluhm, V. Mantovani Sarti, M. Nahrgang, and C. Ratti, Phys. Lett. B 738, 305 (2014).

[42] R. V. Gavai and S. Gupta, Phys. Lett. B 696, 459 (2011).

[43] A. Bazavov, H.-T. Ding, P. Hegde, O. Kaczmarek, F. Karsch, E. Laermann, S. Mukherjee, P. Petreczky, C. Schmidt, D. Smith, W. Soeldner, and M. Wagner, Phys. Rev. Lett. 109, 192302 (2012).

[44] S. Borsányi, Z. Fodor, S. D. Katz, S. Krieg, C. Ratti, and K. K. Szabó, Phys. Rev. Lett. 111, 062005 (2013).

[45] S. Borsanyi, Z. Fodor, S. D. Katz, S. Krieg, C. Ratti, and K. K. Szabo, Phys. Rev. Lett. 113, 052301 (2014).

[46] J. Cleymans, H. Oeschler, K. Redlich, and S. Wheaton, Phys. Rev. C 73, 034905 (2006).

[47] J.-W. Chen, J. Deng, H. Kohyama, and L. Labun, Phys. Rev. D 93, 034037 (2016). 
[48] B. Berdnikov and K. Rajagopal, Phys. Rev. D 61, 105017 (2000).

[49] B. B. Abelev et al. (ALICE Collaboration), Phys. Lett. B 739, 139 (2014).

[50] L. F. Palhares, E. S. Fraga, and T. Kodama, J. Phys. G 38, 085101 (2011).

[51] N. Magdy, M. Csanád, and R. A. Lacey, J. Phys. G 44, 025101 (2017).

[52] G. Y. Shao, L. Chang, Y.X. Liu, and X. L. Wang, Phys. Rev. D 73, 076003 (2006).

[53] A. Bhattacharyya, P. Deb, S. K. Ghosh, R. Ray, and S. Sur, Phys. Rev. D 87, 054009 (2013).

[54] A. Bhattacharyya, R. Ray, and S. Sur, Phys. Rev. D 91, 051501(R) (2015).

[55] A. Bhattacharyya, S. K. Ghosh, R. Ray, K. Saha, and S. Upadhaya, Europhys. Lett. 116, 52001 (2016).

[56] M. S. Berger and R. L. Jaffe, Phys. Rev. C 35, 213 (1987).

[57] D. Deutsch and P. Candelas, Phys. Rev. D 20, 3063 (1979).

[58] H. T. Elze and W. Greiner, Phys. Lett. B 179, 385 (1986).

[59] W. Y. Ke and Y. X. Liu, Phys. Rev. D 89, 074041 (2014).

[60] F. Gao and Y. X. Liu, Phys. Rev. D 94, 094030 (2016).

[61] Y. P. Zhao, R. R. Zhang, H. Zhang, and H. S. Zong, Chin. Phys. C 43, 063101 (2019).

[62] C. D. Roberts and A. G. Williams, Prog. Part. Nucl. Phys. 33, 477 (1994).

[63] C. D. Roberts and S. M. Schmidt, Prog. Part. Nucl. Phys. 45, S1 (2000).

[64] P. Maris and C. D. Roberts, Int. J. Mod. Phys. E 12, 297 (2003).

[65] R. Alkofer, Phys. Rep. 353, 281 (2001).

[66] A. Bashir, L. Chang, I. C. Cloët, B. El-Bennich, Y. X. Liu, C. D. Roberts, and P. C. Tandy, Commun. Theor. Phys. 58, 79 (2012).

[67] I. C. Cloet and C. D. Roberts, Prog. Part. Nucl. Phys. 77, 1 (2014).

[68] L. Chang, Y. X. Liu, and C. D. Roberts, Phys. Rev. Lett. 106, 072001 (2011).
[69] S. X. Qin, L. Chang, Y. X. Liu, C. D. Roberts, and S. M. Schmidt, Phys. Lett. B 722, 384 (2013).

[70] S. X. Qin, L. Chang, Y.X. Liu, C. D. Roberts, and D. J. Wilson, Phys. Rev. C 84, 042202(R) (2011).

[71] S. X. Qin, L. Chang, Y.X. Liu, and C. D. Roberts, Phys. Rev. D 84, 014017 (2011).

[72] C. S. Fischer, Phys. Rev. Lett. 103, 052003 (2009).

[73] C. S. Fischer and J. A. Mueller, Phys. Rev. D 80, 074029 (2009).

[74] E. Gutierrez, A. Ahmad, A. Ayala, A. Bashir, and A. Raya, J. Phys. G 41, 075002 (2014).

[75] B. Wang, Z. F. Cui, W. M. Sun, and H. S. Zong, Few Body Syst. 55, 47 (2014).

[76] K. L. Wang, Y. X. Liu, L. Chang, C. D. Roberts, and S. M. Schmidt, Phys. Rev. D 87, 074038 (2013).

[77] F. Gao and Y. X. Liu, Phys. Rev. D 94, 076009 (2016).

[78] N. Haque, M. G. Mustafa, and M. Strickland, Phys. Rev. D 87, 105007 (2013).

[79] R. Contant and M. Q. Huber, Phys. Rev. D 96, 074002 (2017).

[80] J. Bernhardt, C. S. Fischer, P. Isserstedt, and B.-J. Schaefer, Phys. Rev. D 104, 074035 (2021).

[81] J. Madsen, Phys. Rev. Lett. 85, 4687 (2000).

[82] G. Lugones and A. G. Grunfeld, Phys. Rev. C 103, 035813 (2021).

[83] R.-A. Tripolt, J. Braun, B. Klein, and B.-J. Schaefer, Phys. Rev. D 90, 054012 (2014).

[84] M. S. Abdallah et al. (STAR Collaboration), Phys. Rev. Lett. 127, 262301 (2021).

[85] A. Pandav (STAR Collaboration), Nucl. Phys. A1005, 121936 (2021).

[86] T. Nonaka (STAR Collaboration), Nucl. Phys. A1005, 121882 (2021).

[87] A. Bazavov et al. (HotQCD Collaboration), Phys. Rev. D 101, 074502 (2020).

[88] W. J. Fu, X. Luo, J. M. Pawlowski, F. Rennecke, R. Wen, and S. Yin, Phys. Rev. D 104, 094047 (2021). 